\title{
Group ID
}

National Cancer Institute

\section{Source}

National Cancer Institute. Group ID. NCI Thesaurus. Code C82529.

A sequence of characters that represents a particular group. 